\title{
Flexible polyimide based 34-channel electrode arrays for mouse EEG measurement
}

Fatima Nafisa Chowdhury, Rachit Sood, Hyungwoo Nam, Mary Kay Lobo, Fow-Sen Choa

Fatima Nafisa Chowdhury, Rachit Sood, Hyungwoo Nam, Mary Kay Lobo, Fow-Sen Choa, "Flexible polyimide based 34-channel electrode arrays for mouse EEG measurement," Proc. SPIE 11020, Smart Biomedical and Physiological Sensor Technology XV, 110200T (2 May 2019); doi: $10.1117 / 12.2518830$

Event: SPIE Defense + Commercial Sensing, 2019, Baltimore, Maryland, United States 


\title{
Flexible polyimide based 34-channel electrode arrays for mouse EEG measurement
}

\author{
Fatima Nafisa Chowdhury ${ }^{1}$, Rachit Sood ${ }^{1}$, Hyungwoo $\mathrm{Nam}^{2}$, Mary Kay Lobo ${ }^{2}$, Fow-Sen Choa ${ }^{1}$ \\ ${ }^{1}$ University of Maryland Baltimore County, Computer Science and Electrical Engineering; ${ }^{2}$ University of \\ Maryland Baltimore, School of Medicine
}

\begin{abstract}
Electroencephalogram (EEG) recording is a widely used method to measure electrical activity in the brain. Rodent EEG brain recording not only is noninvasive but also has the advantages to accomplish full brain monitoring, compared with that of the invasive techniques like micro-electrode-arrays. In comparison to other noninvasive recording techniques, EEG is the only technique that can achieve sub-ms scale time resolution, which is essential to obtain causal relationship. In this work, we demonstrated a simple microfabrication process for developing a high-density polyimide-based rodent EEG recording cap. A 34-channel rodent electrode array with a total size of $11 \mathrm{mmx} 8 \mathrm{~mm}$, individual electrode diameter $240 \mu \mathrm{m}$ and interconnect wire linewidth $35 \mu \mathrm{m}$ was designed and fabricated. For the fabrication process, we first deposit $350 \mathrm{~nm}$ $\mathrm{SiO} 2$ on a silicon substrate. We then fabricate 6-7 $\mu \mathrm{m}$ thick first layer polyimide caps with fingers and contact holes. Gold deposition and then lithography etching of 34 channel contact-electrodes and their interconnects were fabricated in the second step. The third step was to cover metal interconnects with a $10 \mu \mathrm{m}$ thick second layer polyimide, which was fabricated with photolithography before the final film released by $\mathrm{HF}$ undercutting etching of $\mathrm{SiO}_{2}$ layer. Then the fabricated EEG cap is interfaced with a commercial 34-channel female connector, which is soldered with 34-line wires. These wires are then connected to an ADC to record the EEG data in computer for post-processing. With polyimide, the EEG cap is biocompatible, and flexible which makes it suitable for good contact with rodent skulls.
\end{abstract}

Keywords: EEG, Polyimide, Micro-Fabrication, Photolithography, EEG Recording cap, Brain activity, Biocompatible, flexible microelectrode.

\section{INTRODUCTION}

Ability to record electrical activity from brain tissues has been of significant interest to researchers who studies brain [13]. Neuron cell is an electrically excitable cell and serves as the primary functional unit of brain tissues. Neurons communicate with each other through electrical signals, known as action potentials or spikes. Reliable monitoring of these nerve impulses is critical to understand the activity pattern in a small or large neuronal network. Electroencephalogram, commonly known as EEG, is a widely used method for recording electrical activity in brain [4]. EEG recording in human brain yields useful spatio-temporal information about brain activities. However, human brain is a significantly complex structure that consists billions of nerve cells and several hundred trillion of connections between them. To understand the brain activity, researchers generally aim to focus on a very specific section of the brain and that requires recording from a much smaller and simpler network of neurons. Rodent animal models are usually chosen for most of these studies since their brains have a simpler architecture than a human brain while it is complex enough to be comparable to activity patterns in a human brain. However, Unlike EEGs for human brain, EEG electrodes for rodent brain need to be significantly smaller in size and this brings few challenges in making reliable EEE electrodes at that scale.

Smart Biomedical and Physiological Sensor Technology XV, edited by Brian M. Cullum,

Douglas Kiehl, Eric S. McLamore, Proc. of SPIE Vol. 11020, 110200T · (c) 2019

SPIE $\cdot$ CCC code: $0277-786 X / 19 / \$ 18 \cdot$ doi: $10.1117 / 12.2518830$

Proc. of SPIE Vol. 11020 110200T-1 
Over the years, there has been a variety of EEG electrodes developed from micro needle-based to pin-based electrodes to record brain signals [1-3, 5-6]. Polyimide based microelectrode is a relatively new approach and it enables the recording of activity at very high spatial and temporal resolution. Some early pioneer works in this direction has investigated this approach and opened the door to new possibilities [7-10]. These reported polyimide-based electrodes have shown the potential for recording activity with high resolution. Polyimide-based electrodes brings the mechanical flexibility in the electrode positioning while it is also biocompatible to be used directly or near to brain surface [11-12].

In our work, we have focused on the design and development of a flexible substrate polyimide-based 34-channel mouse EEG cap. Following our previous works on Kapton film based 8-channel electrode, as shown in Figure 1, increasing the number of electrodes was the next logical step [13-15]. In this paper, we are reporting our design and development of 34channel electrode. In our previous work with 8-channel mouse EEG, we were able to record mouse EEG signals and various cardiac artifacts. To accomplish better accuracy for 2-d to 3-d sLORETA conversions and to employ independent component analysis, we need more channels [14-15]. Apart from increasing the number of electrodes to four times, the fabrication process was significantly different in this work. One of the goals of this work was to develop an EEG cap which is flexible and biocompatible, so that we can do long-term recording from the same region of interest in brain while keeping the recording site minimally impacted. Increasing the number of electrodes offers the opportunity to have better spatial resolution and therefore, allows to move one step closer to the resolution that is relevant to study the brain activity at the neuronal level. With increased number of electrodes for a given surface area, this also brings challenges, such as, risk of overlapping electrode lines, signal crosstalk between adjacent electrodes and complexity in interfacing of this higher number of electrodes to the next section through connection pad. In next few sections, we have discussed the important steps in design and fabrication of this 34-channel, flexible and biocompatible electrode and in last section, briefly outlined the intended application of this electrode as future work.

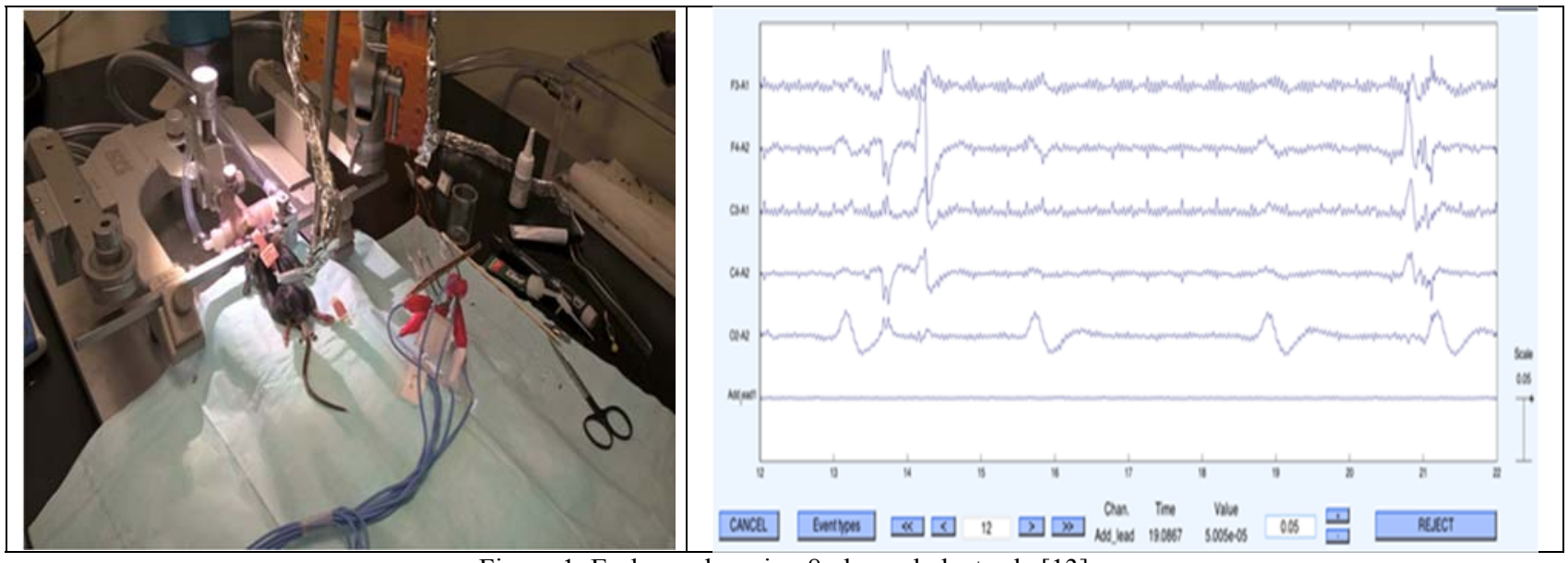

Figure 1. Early works using 8-channel electrode [13]

\section{DESIGN, MATERIALS and FABRICATION}

Building process of the flexible 34-channel electrode consisted of three major phases - designing the electrode mask, selection of materials for different steps of fabrication and then fabricating this device using planned protocols. 


\subsection{Photo Mask Design for 34-channel EEG Electrode:}

To allocate 34 channels in $11 \mathrm{~mm}$ x $8 \mathrm{~mm}$ area, a mask was designed in AutoCAD in such a way so that all the electrode channels are equally spaced. In addition to that, the electrodes were designed to be placed in a tree branch-like architecture where the empty spaces between branches will be useful to put dental cement when it will be placed on mouse skull for EEG recording. As shown in Figure 3, the diameter of each electrodes are 240 micrometers, the conducting line thickness is 35 micrometers. At the rear end, there were 34 connector pads designed each with an area of 250 by 1500 micrometers. These dimension of connector pads ensures an easy connection of the device to the external connector. The other end of connector will be connected to a 15-mm long and wide stripe as shown in Figure 2(d) which is designed for easy handling during recording.

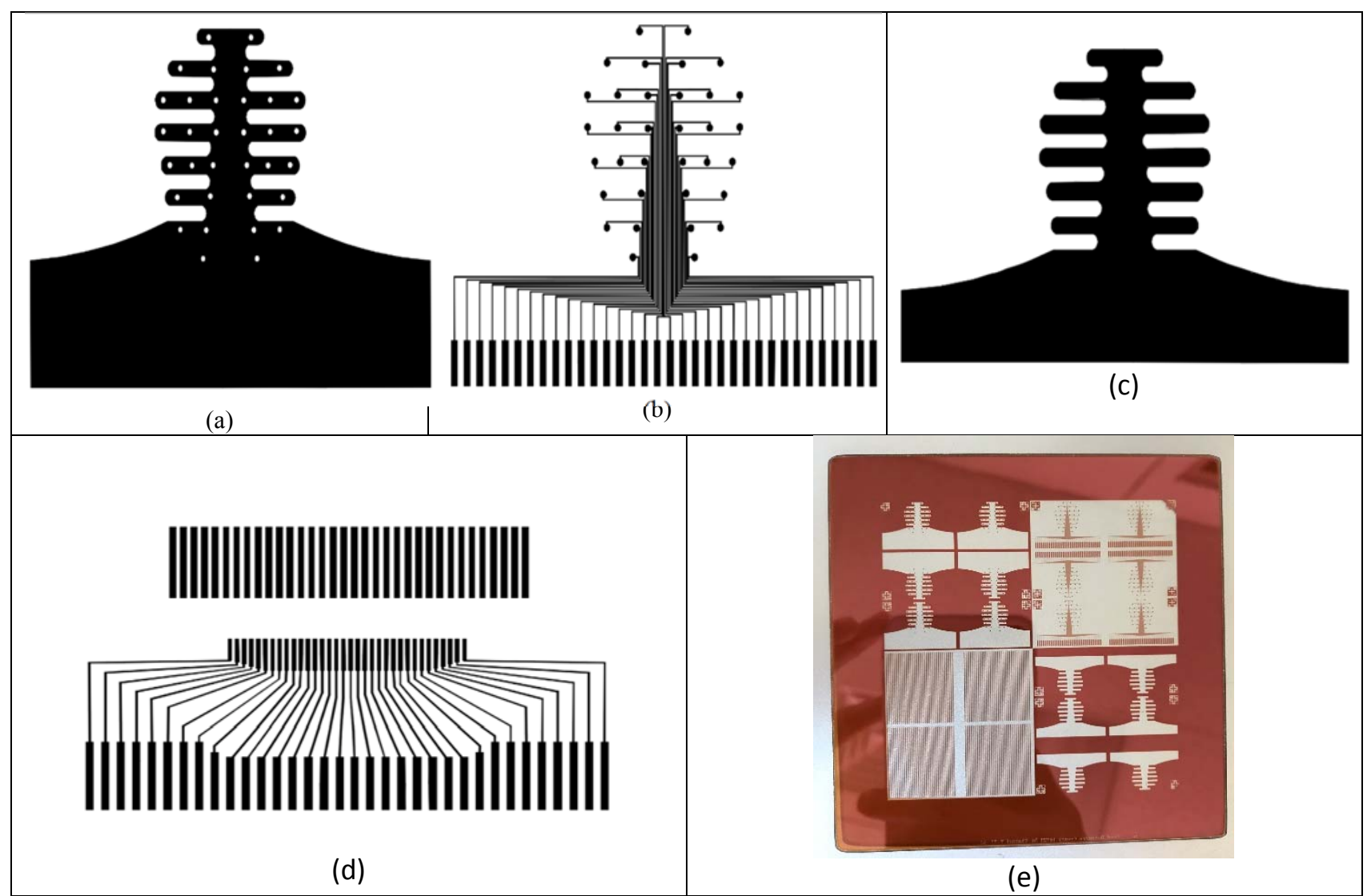

Figure 2. Photo mask design in AutoCAD for 3-Level fabrication. (a): It shows the first layer design with tiny holes in electrode locations. (b): shows the $2^{\text {nd }}$ layer with circular electrodes and connecting lines with them. (c): Last layer for isolation. (d): some designs for external connections. (e): Iron-oxide photo-mask.

\subsection{Materials:}

We have designed our EEG electrode device based on flexible substrate Polyimide, which is a photosensitive Polyimide named Duramide 7510 (Fujifilm). This polyimide is suitable for wide range of application on a wide range of substrates. It is an excellent material for biocompatibility and micro fabrication. The main reason of using polyimide as our base is its flexibility, biocompatibility and its light weight benefit. Also, it is photo-imageable, solvent developable. The other chemicals used were Duramide developer (QZ3501), photoresist AZ5214, AZ400k developer, gold, gold etchant, hydrofluoric acid, Kapton film, ferric chloride. 


\subsection{Fabrication process of 34-channel electrode array:}

Standard photolithographic clean room procedure was followed during fabrication. The fabrication started with a silicon (Si) substrate. First a 350nm thick layer of oxide layer is formed which will be used as a sacrificial layer to release the final device from Si substrate. Then polyimide is spin coated, patterned with UV lithography followed by post baking and developing with QZ3501 developer which formed the bottom layer polyimide cap as shown in figure 3(4). Next, curing it at 350-degree Celsius is performed in nitrogen environment. In next step, a 3000 Angstrom gold layer was sputtered. AZ5201 photoresist was spun and then patterned by UV lithography and developed to generate the metal layer pattern on photoresist. Undesired gold was then etched away, and photoresist was removed using acetone. Thus, a desired metal pattern was left on top of bottom polyimide as in Figure 3(9). Next, a top polyimide layer was formed using the same method of bottom polyimide layer. This layer was to insulate the electrodes and contact lines from top as shown in Figure 3(11). The whole design is then released from silicon wafer and cured in nitrogen environment at 350-degree Celsius for 30 minutes. The final device is shown in Figure 3(12).

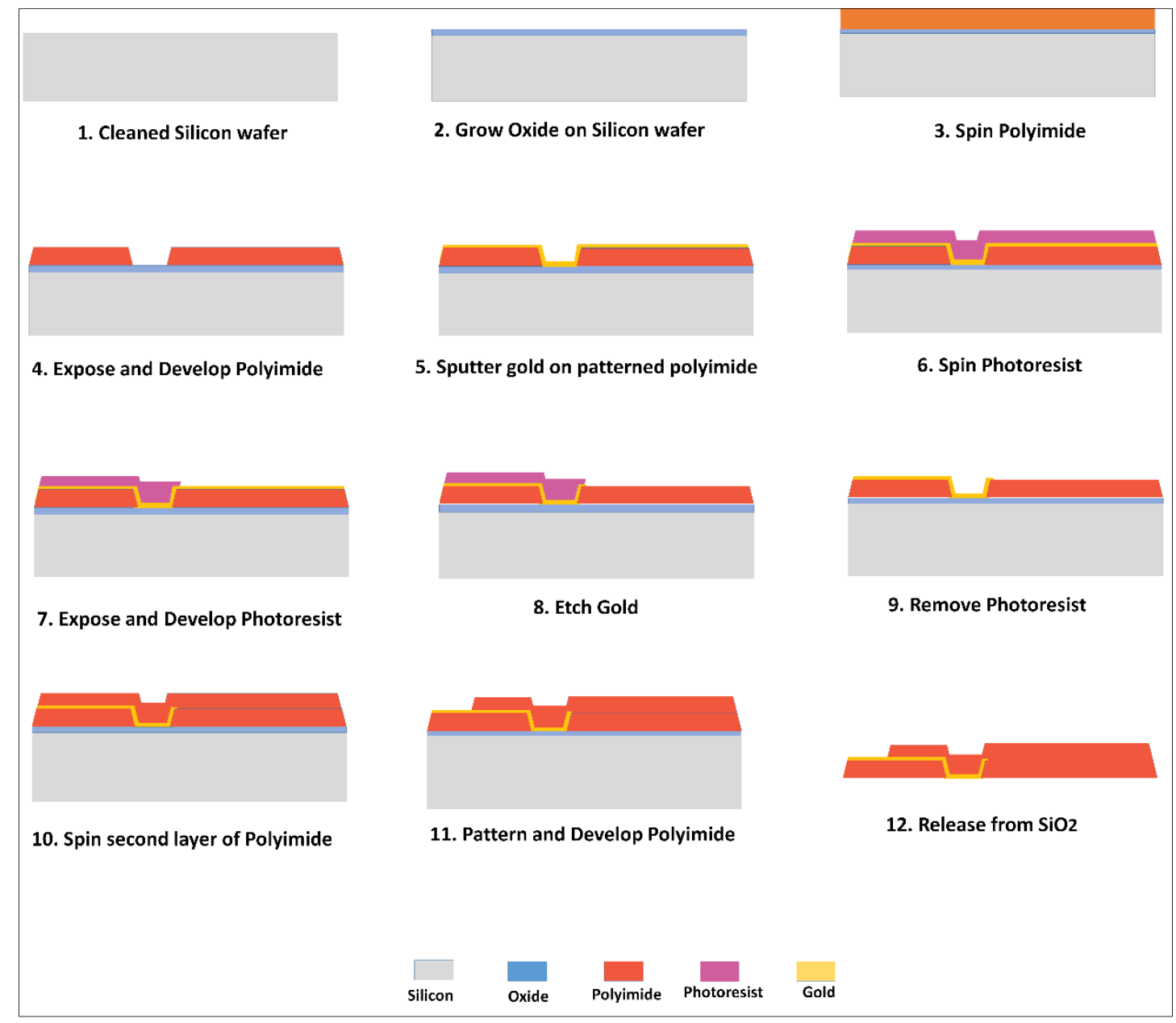

Figure 3: Layer by layer fabrication on Silicon substrate 


\subsection{Interfacing to External Connector}

After completing fabrication, the 34-channel electrode was interfaced with external connector. For making external connections, two lightweight Kapton film was used and then desired pattern were formed by lithography. The Kapton films were patterned using design mask as shown in Figure 2(d) above, which formed desired copper lines. This process was done by spinning the film with photoresist, exposed in UV and developed with AZ400K and finally etching copper with Ferric Chloride $\left(\mathrm{FeCl}_{3)}\right.$. The ends of these patterned contact lines were soldered with connector male pins and the other end with widely separated lines were soldered with wires. Finally, these wires will be connected to an ADC where we will get the desired EEG signal. The external connections were done in such a way so that the external wiring can be removed from the connector, when the device is not being used for recording EEG.

\section{RESULTS and DISCUSSION}

In this section, fabrication results and subsequent testing and validation of the electrodes are briefly described.

\subsection{Successful Fabrication:}

Fabrication was carried out in Technology Research Center Fabrication lab at UMBC, following the steps described in Section 2.2. Final outcomes are shown in Figure 4. The thickness of the final 34 electrode array as shown in figure 4(c) is only 12 micrometers which ensures the reduction of bending stiffness. The length and width are $11 \mathrm{~mm}$ and $8 \mathrm{~mm}$, respectively.

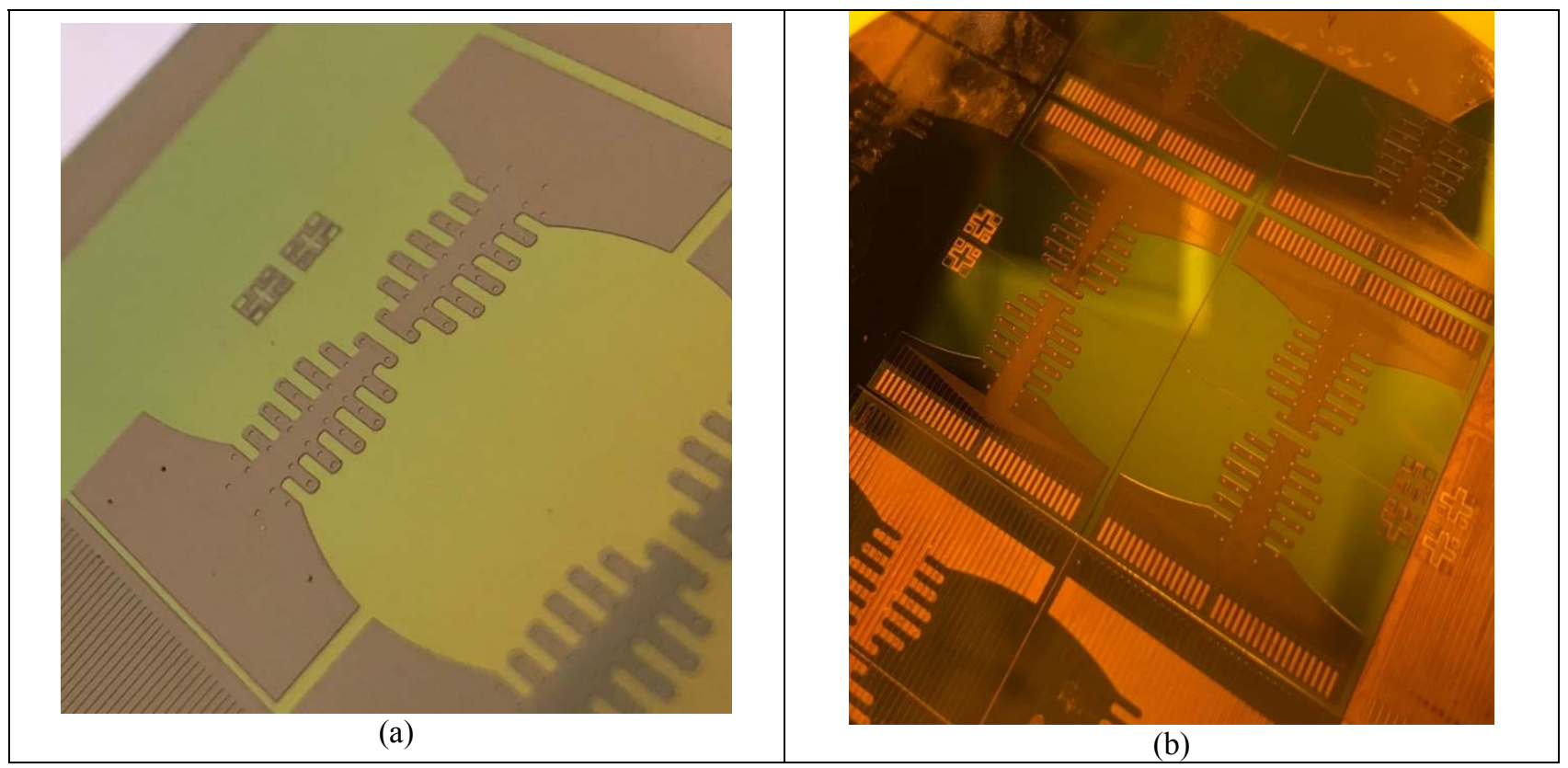




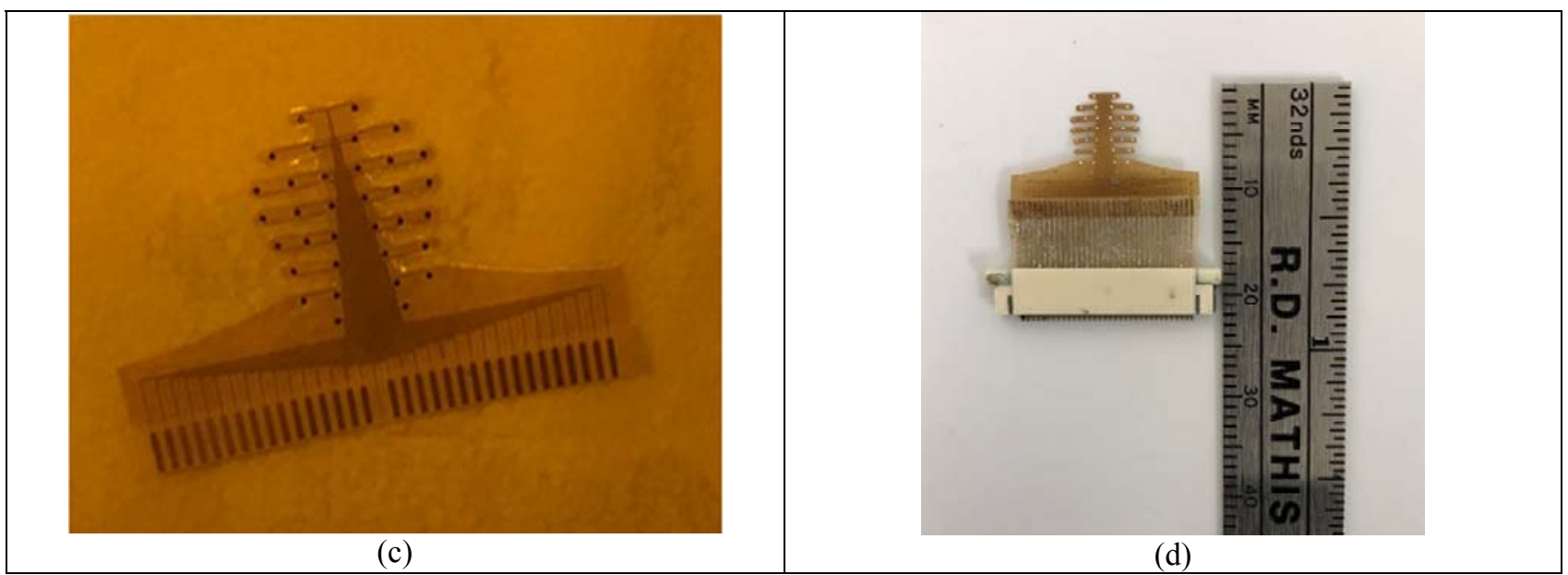

Figure 4. Photograph of metallic mask board and final version of 34-channel electrode. (a) After patterning first polyimide layer (b) Desired pattern of metal and insulating top polyimide layer is formed (c) final product of 34-channel electrode fabrication after lifting it off from substrate (d) Electrode after interfacing, shown with a ruler for physical dimension measurement.

\subsection{Device Testing and Validation}

After the electrode was fabricated, an important step was to validate the quality of connection for all electrodes. A test has been done to determine the connectivity of each electrode. This is done by putting the flexible electrode device on a gold metal plate so that all the electrodes will touch the metal plate, the same way it would be done on mouse. A simple connectivity test in then performed using a Digital MultiMeter (DMM) from the output end (that is the connector pins) which shows that output end is getting signals from input.

Measured resistance for electrodes were ranging between 120-480 Ohms. This was measured between a metal surface that was in contact with all the electrodes and the connector pins on the other side of electrode. The variation in measured resistances is most likely from measurement error due to lack of flexibility in probing very small pins of connector.

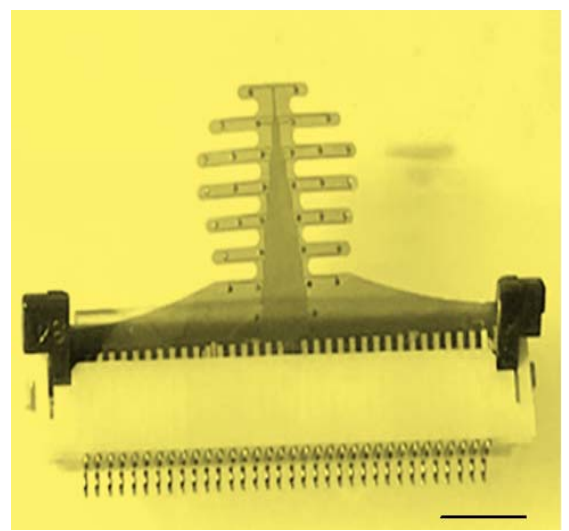

Figure 5. Direct interfacing of 34-channel electrode to a connector. Scale bar: $2.5 \mathrm{~mm}$.

However, for mouse recording application, the above interfacing setup may not be practical. Keeping the relatively heavy weight connector so close to electrode and carrying the load on top of the mouse brain may cause tension to the electrode and there is a risk of getting the device disconnected from the connector. Therefore, we have devised a better approach which is shown in Figure 6. A Kapton film, which went through the process described earlier in Section 2.4 and it has 34 straight lines that is designed to conduct the signal from one end of film to the other end, is inserted between the electrode 
and connector. The top end of the film is to receive the endpoints of electrode, either soldered or tightly taped, and then the other end is inserted into the connector, as shown in Figure 6. This will ensure the connector is safely at a distance from mouse brain recording site.

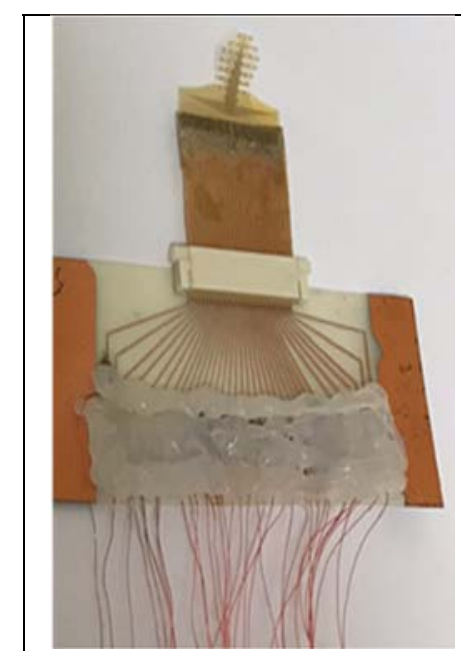

(a)

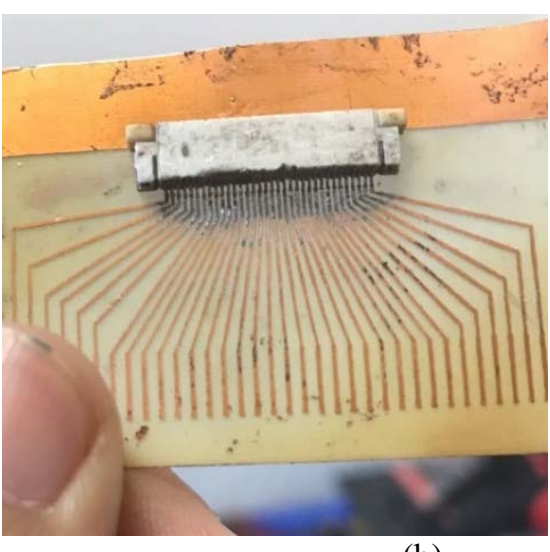

(b)

Figure 6. Interfacing of 34-channel electrode via a Kapton film to connector and subsequent soldered wires.

\section{CONCLUSION AND FUTURE WORK}

In this work, a polyimide-based flexible 34-channel electrode was designed using CAD and then implemented its fabrication following step-by-step process. After fabrication, electrode was interfaced with 34-channel connector and then validated the channel quality for signal propagation. The data acquisition system was also setup to acquire the signals from this electrode and to execute subsequent analysis and post-processing. Next step of this work is to carefully install this electrode assembly on top of mouse model in vivo and then record EEG signals. We believe that increased spatial resolution of electrode channels in this new device will allow us to record more meaningful and robust information from brain surface and therefore, will improve our understanding of brain activity.

\section{REFERENCES}

1. Duffy, F. H., Burchfiel, J. L. and Lombroso, C. T. (1979), Brain electrical activity mapping (BEAM): A method for extending the clinical utility of EEG and evoked potential data. Ann Neurol., 5: 309-321.

2. D. Kim, C. Yeon, E. Chung and K. Kim, "A non-invasive flexible multi-channel electrode for in vivo mouse EEG recording," SENSORS, 2014 IEEE, Valencia, (2014) 
3. Wasilczuk, A.Z., Proekt, A., Kelz, M.B., McKinstry-Wu, A.R. High-density Electroencephalographic Acquisition in a Rodent Model Using Low-cost and Open-source Resources. J. Vis. Exp. (117) (2016)

4. E. Niedermeyer, F. H. Lopes da Silva. Electroencephalography: Basic principles, clinical applications and related fields, 3rd edition (1993)

5. Jiahui Wang, Zhuolin Xiang, Gil Gerald Lasam Gammada, Nitish V. Thakora,b, Shih-Cheng Yen, Chengkuo Lee. Development of flexible multi-channel muscle interfaces with advanced sensing function, Sensors and Actuators, 249, (2016)

6. T Frederik Ceyssensa, Marta Bovet Carmonab, Dries Kila, Marjolijn Deprezc, Ester Tootend, Bart Nuttinc, Aya Takeokae, Detlef Balschunb, Michael Krafta, Robert Puersa; Chronic neural recording with probes of subcellular cross-section using $0.06 \mathrm{~mm}^{2}$ dissolving microneedles as insertion device. Sensors and Actuators B: Chemicals $284,(2019)$

7. Chung, J. E. et al. High-Density, Long-Lasting, and Multi-region Electrophysiological Recordings Using Polymer Electrode Arrays. Neuron 101, 21-31, (2019)

8. Richardson R Jr, Miller J, Reichert W (1993) Polyimides as biomaterials: preliminary biocompatibility testing. Biomaterials 14(8):627

9. Ghosh M, Mittal K (1996) Polyimides: fundamentals and applications. Marcel Dekker, New York, p 367

10. Natàlia de la Oliva, Xavier Navarro, Jaume del Valle "Time course study of long term biocompatibility and foreign body reaction to intraneural polyimide based implants" Journal of Biomedical Materials Research (2017)

11. Choi JH, Koch KP, Poppendieck W, Lee M, Shin HS. High resolution Electroencephalography in Freely Moving Mice. J Neurophysiol (2010).

12. Lee M, Shin HS, Choi JH. Simultaneous recording of brain activity and functional connectivity in the mouse brain. Conf Proc IEEE Eng Med Biol Soc. (2009)

13. D. Gupta, M. Islam, H. Nam, M. K. Lobo, F.-S. Choa, "Kapton polyimide-based EEG microelectrode array and interfaces for mice brainwave recordings and analysis" Proceedings of SPIE (2018)

14. Qinglei Meng, Deepa Gupta, Abenezer Wudenhe, Xiaoming Du, L. Elliot Hong, Fow-Sen Choa, "Threedimensional EEG signal tracking for reproducible monitoring of self-contemplating imagination", Adv. Sci. Technol. Eng. Syst. J. 2(3), 1634-1646 (2017);

15. Gupta D, Du X., Hong L., Choa F., "TMS-EEG based Source Localized Connectivity Signature Extraction by using Unsupervised Machine Learning”, 9th International IEEE/EMBS Conference on Neural Engineering (NER), March 2019, Sam Francisco CA. 